\title{
Research on Key Technologies of Computer Graphics Image Processing
}

\author{
Ying Zhou, Pancheng Li \\ Jiangxi Police College, Nanchang, 437100, China
}

Keywords: Computer Graphics Image, Image Processing, Key Technology

\begin{abstract}
With the continuous development of science and technology, computer technology has also made great progress. Graphic image processing technology is an important part of computer technology, and it is also widely used in modern application science. Computer graphics and image processing technology brings a lot of convenience to people's daily life, work, and learning, and it also has a strong impact on people's vision. The article discusses computer graphics and computer graphics image processing technologies, analyzes the differences between computer graphics and image technologies, and discusses the practical application of key technologies of computer graphics and image processing in order to promote the orderly health of computer graphics and image processing technologies to provide some ideas.
\end{abstract}

\section{Introduction}

In the 1980s, computer graphics and image processing technology emerged as a new industry. It is a science and technology that uses computers to design, adjust, and beautify graphic images. Computer graphics and image processing technologies include CAD, CAE and many other application softwares, and their development prospects are very considerable. In addition, computer graphic image processing technology has been widely promoted, not only brings more convenience to people's daily life, study and work, but also promotes the development of related industries. Therefore, it is very important to study computer graphics and image processing technology. Meaning.

\section{Contents of computer image processing technology}

The application principle of computer image processing technology is very simple. It is a process of preprocessing the image through optics. The consolidation of digital technology after optical processing makes the processing more accurate, and the combination of these two technologies is used to enhance the precision and rationalization of the image. The electric mode processing is generally more common in television equipment applications [1]. It is mainly a process that uses the conversion between the optical signal and the electrical signal to analyze and analyze the signal by using electronics. The continuous development of science and technology is also a breakthrough and improvement in the handling of electricity patterns. It is mainly to realize the function of identifying and scanning images and find out the information contained in the images and the specific content. For example, fingerprint recognition and face recognition are practical applications of image recognition technology. The important components of computer image processing technology are image restoration and enhancement technology. The main purpose is to improve the clarity of the image. The principle is to improve the contrast of the image, change the size of the image, and optimize the image. The computer's image processing works by transforming image information such as photos and drawings into computer-recognizable information during storage. Information is processed through computers to obtain the information we want. The digitization of images mainly includes sub-sampling and quantization as well as coding. Sampling is based on the number of points in the image. The quality of the sample depends mainly on the resolution of the image. The more times the sample is taken, the higher the accuracy of the display. The space that is used is also the memory. Determine the range of data used for sampling to indicate that the total number of image colors plays a decisive role. In order to facilitate the use of pictures in life, and the 
number of images after processing is increasing, more images are required to be compressed and saved. This requires the use of compression coding techniques. Its main purpose is to reduce the memory lock occupied by the image, save the image, and effectively compress the image.

\section{Overview of Computer graphics image processing technology}

Computer graphics and image processing technology refers to achieving high-speed and high-efficiency processing of graphic images with the aid of powerful computer operating levels. At this stage, there are many different types of computer graphics image processing technologies, and each function is different. In recent years, various fields generally combine dimensional image processing software and processing systems into two-dimensional graphic image processing technologies and three-dimensional graphic image processing technologies in combination with dimensional differences. The former processing technology emphasizes color design through paired graphic images. The restoration, enhancement, and segmentation operations accomplish the processing of the graphic image; the latter processing technique emphasizes the scaling, perspective, and projection of the image, and has more processing and processing capabilities for the three-dimensional relationship of the graphic image. During the development of graphic image processing, there are times when the two graphic image processing technologies should be effectively combined, so that the graphic image processing technology can be used to the optimal level, thereby providing more favorable support for production and living [2].

Based on morphological mathematics and three-dimensional mathematics, computer graphics technology is formed. Therefore, in the process of processing related graphics and images, the first step is to digitize graphics and images, and then turn them into The computer-recognized format is described by taking the computer image processing technology as an example. Before the computer image processing, sampling is first performed. After the sampling work is completed, the image is divided in the vertical direction or the horizontal direction, and then the captured image is acquired. The data points perform specific quantification work, and then the coding is compressed. The coding and compression link is an important step for storing computer images. Predictive coding, transform coding, wave point change compression coding, etc. are currently more widely used coding compression methods.

The relevant professional technicians developed a software using image processing equipment. This is CAD technology. The software is now widely used in the design of enterprise products. Staff can use this software to more easily store important information and draw graphs. When using CAD technology to correlate computer images, face different design schemes, treat them differently, make in-depth and detailed analysis and research, and finally choose the best design scheme and use the corresponding functions of the software to handle them. Graphical information and textual information are searched and retrieved to enable editing and moving of graphics and texts. According to relevant personnel, the use of CAD technology has involved mechanical design, geological exploration, software development, apparel design and many other industries and different fields. The main technical content of CAD is interactive technology, surface modeling technology, and interactive technology [3]. It is able to use the coordinate system established by the user to output the display device through the graphics, realize the design editing work such as rotation, perspective, and translation of the graphics or image information, and eventually achieve the graphic change. Final goal. At the same time, interaction technology, as an indispensable processing technology in the computer field, can help humans easily and intuitively complete human-machine information exchange in the design work. In this way, it can realize the virtual work of the designer, data correction, and other preparatory work, and more importantly. One of the functions is to present the result of the design or modification work in an instant or in a short time, which greatly improves the work efficiency and design accuracy of the relevant personnel. CAM technology belongs to a class of computer manufacturing process and first appeared in Guan country. The purpose of this program is to enable the control and management of related products. With the rapid development of computer technology, at this stage, CAM has achieved a geometric definition. The application of language, can carry on certain optimization to the image or figure 
information or program preparation, batch processing. Because of the salient features and strong advantages of CAM, many industries have now introduced CAM and have achieved very good results. As one of the mainstream technologies in the computer field, graphic processing has been developed for a long time and has also been relatively complete. In addition to its own practical enhancement, this technology has been widely applied and popularized [4].

\section{Application of Graphic Image Processing Technology}

With the continuous development of social development and science and technology, graphic image processing technology in the computer field has long been widely applied to all aspects of their lives and work. The application of this technology is mainly reflected in the auxiliary design, auxiliary manufacturing, etc., and has the powerful advantages of intuitive and timely visualization, among which CAD is one of the more widely used technologies. In the industry, CAD technology can help people to analyze and store graphic image data, which is bound to become one of the important tools for technology promotion and application. In the research and development of industrial products, related personnel use the form of parameters to input the R\&D program into the computer. Through the help of computer-related software, this will not only provide guidance for the R\&D process, but also further improve product design, production, and quality inspection. Monitor and provide strong guarantee for high quality product design. For example, in the part design of the auto parts industry, as long as the physical test can be used to obtain a wealth of information, scientific analysis and systematic research of the experimental results are provided. Use this method to reduce the cost of manpower and material resources, and at the same time improve the design efficiency of parts and the improvement of the passing rate of experimental results [5]. Therefore, the relevant designers should be used for innovation and continuously improve their overall quality. During product development, developers are required to input the steps of the R\&D process into the computer in the form of parameters. On the one hand, they can provide guidance for the R\&D process. On the other hand, they can monitor product design quality and shape, and thus provide favorable performance for designing and producing products. Protection. By introducing a computer graphics platform, not only can the work efficiency of designers be greatly improved, but also product quality can be improved. For example, in the process of designing automotive parts, the traditional auto parts testing method usually requires actual items. Only by conducting physical tests can the experimental results be obtained and the results analyzed. This kind of test method will obviously cause unnecessary waste of human and material resources. The same experimental results are not necessarily very accurate. By adopting digital methods to develop automotive parts design, it can significantly improve the work efficiency of designers and provide favorable guarantees for product design accuracy. At the same time, with the help of CAD and other simulation of the experimental process, and in the process of assessing automotive control systems such as automotive parts safety performance, structural strength, etc., a computer-aided control system is usually introduced for analysis. This type of system can present system operation modes in the form of graphic images, further ensuring that the system operation can be grasped by more users.

\section{Conclusion}

The rapid development of computer technology has led to a qualitative leap in computer graphics technology. At the same time, it has realized the promotion and application of computer graphics technology in many fields, and brought great convenience to the production and design process of the masses. The promotion has played a powerful role in promoting. Relevant technical personnel should further study and study, earnestly sum up experience, have a clear and profound understanding of the connotation of computer graphics and image technology, continuously improve the application of key technologies of computer graphics and image processing in specific practices, and promote the advancement of computer graphics and image processing technology. Healthy and orderly development. 


\section{References}

[1] Chen Minya, Jin Xudong. Computer graphics and graphic image processing technology [J]. Journal of Changchun University of Science and Technology, 2011 (01): 142-143,150.

[2] Lin Lihong. Discussion on Computer Graphic Processing Technology [J]. China Hi-tech Enterprise, 2015(15):61-62.

[3] Liu Zhuo, Ya Li, Li Dong, Computer graphics, image processing, and related technology exploration [J]. Engineering Technology: quotation, 2016, (26): 11-12.

[4] Zhang Yanhua. Key Technology of Computer Graphics Image Processing [J]. Electronic Technology and Software Engineering, 2017(3).

[5] Song Linlin. Discussion on Key Technologies of Computer Graphics Image Processing [J]. Youth Years, 2014 (19) 